\title{
Prioritizing continuity in Canadian clinical teaching units
}

$\mathrm{T}$ he general internal medicine inpatient teaching service, frequently called the clinical teaching unit (CTU) in Canada, is one of the first places where a medical student or resident takes full responsibility for patients - where he or she is the patient's doctor. It has been the cornerstone of medical education, not just for internists, but for all doctors. Over the past decade, external forces described in a related article in $C M A J^{1}$ have had a dramatic impact on the learning experience in CTUs in Canada. Currently, in addition to working fewer hours than before, students and residents participate in nonCTU-related educational activities (e.g., academic half days) to enhance their skills; these activities divide their focus and require routine absences from the CTU during the rotation. Organizational responses to these challenges have had adverse consequences. We propose an alternative that we believe is better.

Although there is some variation across Canada, we have observed that attending physicians, who set the teams' philosophy, allow senior residents to give priority to coverage: every patient on the roster is seen by a team member every day, regardless of whether that member knows the patient or not. Teams of physicians "run the list" of patients each morning, ask which team members are present and assign each patient to an available resident or student for the day. Trainees are free to participate in their other activities without simultaneously bearing responsibility for inpatient care, and the workload can be evenly distributed every day. However, because this means patients may be cared for by several doctors during their admission, the opportunity to teach the core attributes of continuity of care and long-term responsibility is lost.

Our way of organizing care in the CTU gives priority to continuity rather than coverage. Patients remain under the care of the trainee who admitted them (or to whom they were initially assigned if admitted overnight by a

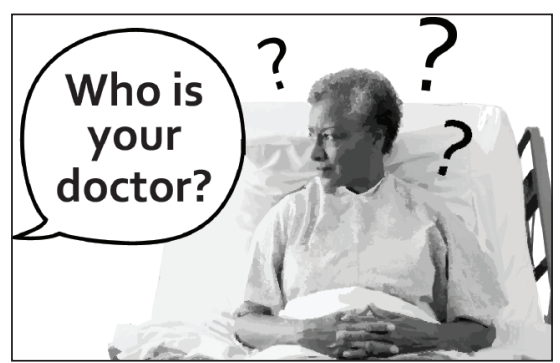

non-team member) for their entire stay. Regardless of other commitments, students and residents maintain responsibility for the assessment and management of their patients every working day: they see their patients, review laboratory and imaging results, interact with consultants, work with allied health professionals and write the daily progress notes. When they have a halfday commitment outside the CTU, they see their patients either before or after the activity. If one of their patients requires an action during their absence, they ask another team member to perform the function but are responsible for ensuring that it is completed. After call, they see their patients on rounds in the morning before reviewing new patients with the team. The only times when trainees do not maintain responsibility for their patients are during weekends off call and vacation. When the workload is uneven, some redistribution of patients may be required.

Giving students and residents the responsibility of solving the problem that prompted the admission, without the option of deferring complex issues to a day when the patient can be assigned to someone else, has a profound effect on their attitude. Having challenging conversations about goals of care or code status, following up on tests ordered and managing adverse effects of therapies are the responsibility of a single person who "wears" the consequences of the clinical decisions he or she makes. Students and residents are motivated to explore the literature and seek the advice of experts about the full arc of their patients' illnesses, not just issues that occur on a given day. Our system is also efficient because trainees do not have to acquaint themselves with a new roster of patients every day. They understand the thread of clinical thinking underlying their patient's care plan because they initiated it.

For our approach to work, the attending physician must establish and prioritize the philosophy of "one patient, one doctor" on the first day of the rotation. This task cannot be delegated to the senior resident, although the cooperation of residents is paramount. In our experience, once the philosophy is established, most trainees understand the benefits to themselves of a system that trains them to be doctors, not "firefighters." They are less likely to utter the dreaded statement often heard in teaching hospitals across Canada: "I don't really know this patient well. I'm just covering for today." Finally, because patients see the same team member every day, they have a much better chance of being able to answer the question, "Who is your doctor?"

Although the benefits and drawbacks of nonclinical duties and restrictions on duty hours continue to be a source of debate, we are not advocating turning back the clock. Instead, we propose a refocusing of priorities in the organization of CTUs to teach future physicians how to take responsibility for the ongoing care they provide. ${ }^{2}$

\section{Shlok Gupta MD}

Allan S. Detsky MD PhD

Institute of Health Policy Management and Evaluation

Department of Medicine

University of Toronto

Toronto, Ont.

\section{References}

1. Pattani R, Wu PE, Dhalla IA. Resident duty hours in Canada: past, present and future. CMAJ 2014; 186:761-5.

2. Detsky AS, Berwick DM. Teaching physicians to care amid chaos. JAMA 2013;309:987-8.

Acknowledgements: The authors thank Drs. Robert Sargeant, Steven Shadowitz and Christopher Smith for their helpful comments on an earlier draft.

CMAJ 2014. DOI:10.1503/cmaj.140565

All editorial matter in CMAJ represents the opinions of the authors and not necessarily those of the Canadian Medical Association. 